\title{
Should optics students know statistics?
}

\section{Anna Consortini}

Anna Consortini, "Should optics students know statistics?," Proc. SPIE 3831, Sixth International Conference on Education and Training in Optics and Photonics, (16 June 2000); doi: 10.1117/12.388745

EPIEnt: Education and Training in Optics and Photonics (ETOP'99), 1999, Cancun, Mexico 


\title{
Should optics students know statistics?
}

\author{
Anna Consortini \\ Physics Department, University of Florence \\ via S.Marta 3, 50139 Florence, Italy ${ }^{1}$
}

\begin{abstract}
Optics students' need for statistical knowledge is investigated. By statistics I mean both probability and statistics. By starting with a simple interferometer some different cases are presented where, from no need for statistical knowledge, one reaches cases where simple probability is needed and finally cases that require profound knowledge of statistics.
\end{abstract}

\section{INTRODUCTION}

The title's question arises from the need to introduce optics students to "non deterministic" optics problems and is aimed to show them a "statistical approach". As already stated in previous papers 1,2 in books of this series, by "optics students" I mean university undergraduates and graduates. In addition, with the word "statistics" I mean both probability and statistics.

To illustrate some ideas and try to find answers to the question, let consider a simple and well known example: the "classic" Young interferometer, namely a screen with two (small) holes or slits. The question whether statistics is needed in studying the behaviour of this interferometer has the answer "it depends". It depends on the field, or fields, illuminating the holes.

The Young interferometer can be presented to the students in a completely deterministic way and this is the way one generally presents interference phenomena for the first time. One assumes that a "monochromatic wave" (that is a perfectly coherent field) impinges on the screen and, from the Huygens-Fresnel principle, produces two spherical waves emerging from the holes. With reference to Fig 1 , let us denote by $v_{1}$ and $v_{2}$ one component of the field at the "output" of the two holes 1 and 2 respectively. As usual we use the scalar approximation (optics approximation), according to which one component of the field describes the entire phenomenon. We also use complex notation and recall that the energy density is proportional to the modulus square of this component. According to common use in optics we use the -i factor. At the holes one has

$$
\begin{aligned}
& \mathrm{v}_{1}=\mathcal{A}_{1} e^{i \varphi_{1}} \mathrm{e}^{-\mathrm{i} \omega \mathrm{t}} \\
& \mathrm{v}_{2}=\mathcal{A}_{2} e^{i \varphi_{2}} \mathrm{e}^{-\mathrm{i} \omega \mathrm{t}}
\end{aligned}
$$

where the amplitudes, $\mathcal{A}_{1}$ and $\mathcal{A}_{2}$, and the phases, $\varphi_{1}$ and $\varphi_{2}$, depend on the field impinging on the screen with holes. For an impinging plane wave, for instance $\mathcal{A}_{1}$ and $\mathcal{A}_{2}$ are equal, while the phases are equal if the wave impinges normally.

At a very far distance from the holes the two spherical waves emerging from the holes can be approximated by two plane waves, so that the two fields $v_{1}(P)$ and $v_{2}(P)$, at point $P$, are given by:

\footnotetext{
${ }^{1}$ tel +39-055-4796415 fax +39-055-483750 e-mail consortini@dffs.unifi.it
} 


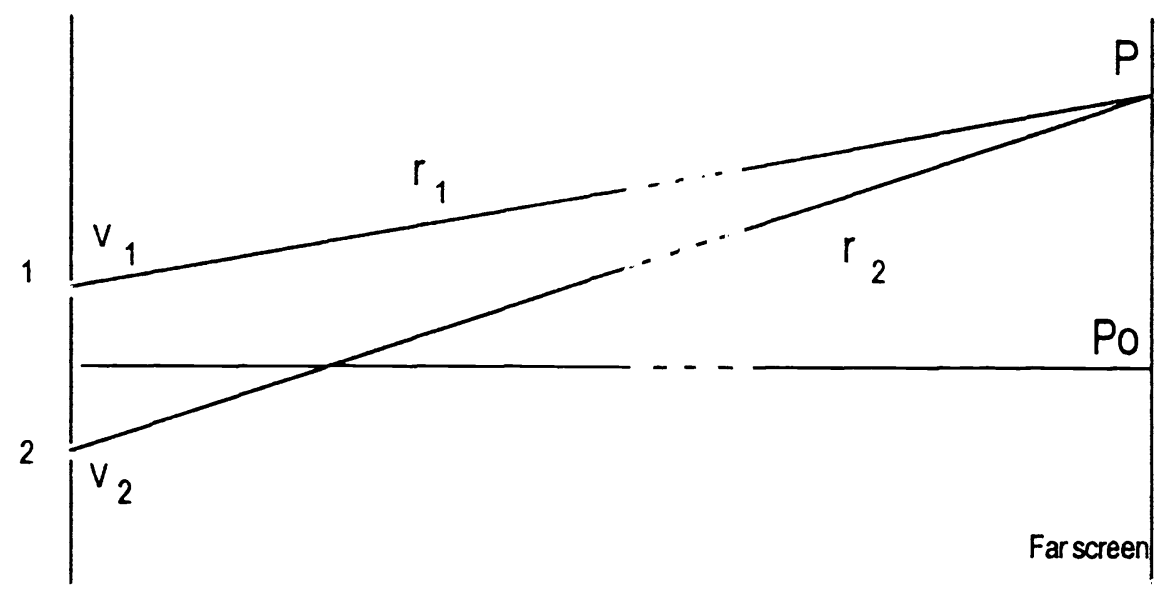

Figure 1

$$
\begin{aligned}
& v_{1}(P)=A_{1} e^{i \varphi} e^{-i\left(\omega t-k r_{1}\right)} \\
& v_{2}(P)=A_{2} e^{i \varphi} e^{-i\left(\omega t-k r_{2}\right)}
\end{aligned}
$$

where amplitudes $A_{1}$ and $A_{2}$ are not necessarily equal to those, $\mathcal{A}_{1}$ and $\mathcal{A}_{2}$, at the holes. The total field $v(P)=v_{1}(P)+v_{2}(P)$ at point $P$ on the far screen is therefore:

$$
v(P)=A_{1} e^{i \varphi} 1 e^{-i\left(\omega t-k r_{1}\right)}+A_{2} e^{i \varphi} 2 e^{-i\left(\omega t-k r_{2}\right)}
$$

The intensity at $\mathrm{P}$ is obtained by multiplying $\mathrm{v}(\mathrm{P})$ by its complex conjugate

$$
\mathrm{I}(\mathrm{P})=\mathrm{A}_{1}^{2}+\mathrm{A}_{2}^{2}+\mathrm{A}_{1} \mathrm{~A}_{2} \mathrm{e}^{\mathrm{i}\left[\varphi_{1}-\varphi_{2}+\mathrm{k}\left(\mathrm{r}_{1}-\mathrm{r}_{2}\right)\right]}+\mathrm{A}_{1} \mathrm{~A}_{2} \mathrm{e}^{-\mathrm{i}\left[\varphi_{1}-\varphi_{2}+\mathrm{k}\left(\mathrm{r}_{1}-\mathrm{r}_{2}\right)\right]}
$$

from which one gets

$$
\mathrm{I}(\mathrm{P})=\mathrm{A}_{1}^{2}+\mathrm{A}_{2}{ }^{2}+2 \mathrm{~A}_{1} \mathrm{~A}_{2} \cos \left[\varphi_{1}-\varphi_{2}+\mathrm{k}\left(\mathrm{r}_{1}-\mathrm{r}_{2}\right)\right]
$$

This is the well known interference pattern on the far screen; the positions of the maxima corresponding to 'argument of $\cos$ ' $=2 n \pi$ and minima to 'argument of $\cos$ ' $=(2 n+1) \pi$.

For impinging plane wave $\left(A_{1}=A_{2}=A\right)$ the intensity in the maxima is 4 times that due to a single hole and in the minima is zero. If in addition the phases at the holes are equal $\varphi_{1}=\varphi_{2}$ (wave impinging normally) at point Po on the axis the intensity is maximum. In the case of plane wave and normal incidence, Eq 7 can be rewritten as

$$
I(P)=2 A^{2}\left\{1+\cos \left[k\left(r_{2}-r_{1}\right)\right]\right\}
$$

To go further however we will keep the more general form given by Eq.7, which constitutes the basis for introducing variability. From now on Eq 7 will be referred to as "mother equation".

\section{A PROBLEM REgUIRING A STATISTICAL APPROACH}

Let us now consider another simple problem: the phase of the field impinging on the holes has small random fluctuations, while the amplitude is still constant. This is a common case arising when a plane wave propagates through an atmospheric path of a few meters. As a consequence, the 
intensity at point $\mathrm{P}$ also undergoes random fluctuations. It is no longer a deterministic quantity but has the behaviour of a statistical quantity and a statistical approach is necessary. Speaking of intensity at point $P$, one could speak of instantaneous value of the intensity or, more usefully, of "average intensity". In addition, if we want to know how much the intensity fluctuates, another important quantity to consider is the "intensity variance".

By starting from the "mother equation" and averaging both sides one can get the average value $<\mathrm{I}(\mathrm{P})>$ of $\mathrm{I}(\mathrm{P})$, where as usual brackets denote average:

$$
<\mathrm{I}(\mathrm{P})>=\mathrm{A}_{1}^{2}+\mathrm{A}_{2}^{2}+2 \mathrm{~A}_{1} \mathrm{~A}_{2}<\cos \left[\varphi_{1}-\varphi_{2}+\mathrm{k}\left(\mathrm{r}_{1}-\mathrm{r}_{2}\right)\right]>
$$

Here use has already made of the property that the average of a sum is the sum of the averages. It is clear that the average value of the $\operatorname{cosine}, \quad\left\langle\cos \left[\varphi_{1}-\varphi_{2}+\mathrm{k}\left(\mathrm{r}_{1}-\mathrm{r}_{2}\right)\right]\right\rangle$, will determine the behaviour of the average intensity pattern. One can write:

$$
\begin{aligned}
& <\cos \left[\varphi_{1}-\varphi_{2}+\mathrm{k}\left(\mathrm{r}_{1}-\mathrm{r}_{2}\right)\right]>= \\
& <\cos \left(\varphi_{1}-\varphi_{2}\right)>\cos \left[\mathrm{k}\left(\mathrm{r}_{1}-\mathrm{r}_{2}\right)\right]-<\sin \left(\varphi_{1}-\varphi_{2}\right)>\sin \left[\mathrm{k}\left(\mathrm{r}_{1}-\mathrm{r}_{2}\right)\right]
\end{aligned}
$$

Let us now make the simplifying assumption that the two phases fluctuate independently with a given probability distribution. By expanding the cosine and sine and taking into account the property that the average of a product of independent variables is the product of the averages, one obtains:

$$
\begin{aligned}
< & \cos \left[\varphi_{1}-\varphi_{2}+\mathrm{k}\left(\mathrm{r}_{1}-\mathrm{r}_{2}\right)\right]>= \\
=\{ & \left\{\cos \varphi_{1}><\cos \varphi_{2}>+<\sin \varphi_{1}><\sin \varphi_{2}>\right\} \cos \left[\mathrm{k}\left(\mathrm{r}_{1}-\mathrm{r}_{2}\right)\right]- \\
& \left\{<\sin \varphi_{1}><\cos \varphi_{2}>-<\cos \varphi_{1}><\sin \varphi_{2}>\right\} \sin \left[\mathrm{k}\left(\mathrm{r}_{1}-\mathrm{r}_{2}\right)\right]
\end{aligned}
$$

To proceed further we now need to know the probability density functions $\mathrm{p}\left(\varphi_{1}\right)$ and $\mathrm{p}\left(\varphi_{2}\right)$ of the phases. First let us assume that they both follow the same distribution $p(\varphi)$. A simple example is a uniform distribution (around zero) in the interval $-\Omega$ to $+\Omega$

$$
\begin{array}{llrl}
\mathrm{p}(\varphi)=1 /(2 \Omega) & \text { for } & -\Omega=<\varphi<\Omega \\
\mathrm{p}(\varphi)=0 & \text { for } & \varphi<-\Omega \text { and } \varphi>\Omega
\end{array}
$$

Note that symmetric probability of the phase centered on zero means $\left\langle\varphi_{1}\right\rangle=\left\langle\varphi_{2}\right\rangle=0$. From the definition of "average" one immediately obtains:

$$
\begin{aligned}
& <\cos \varphi_{1}>=<\cos \varphi_{2}>=(\sin \Omega) / \Omega \\
& <\sin \varphi_{1}>=<\sin \varphi_{2}>=0
\end{aligned}
$$

Introduction of Eq.13 and 14 into 11 and then substitution into Eq.9 gives

$$
<\mathrm{I}(\mathrm{P})>=\mathrm{A}_{1}{ }^{2}+\mathrm{A}_{2}{ }^{2}+2 \mathrm{~A}_{1} \mathrm{~A}_{2} \cos \left[\mathrm{k}\left(\mathrm{r}_{1}-\mathrm{r}_{2}\right)\right](\sin \Omega)^{2} / \Omega^{2}
$$

In the case of equal amplitudes Eq. 15 becomes:

$$
<\mathrm{I}(\mathrm{P})\rangle=2 \mathrm{~A}^{2}\left\{1+\cos \left[\mathrm{k}\left(\mathrm{r}_{1}-\mathrm{r}_{2}\right)\right](\sin \Omega)^{2} / \Omega^{2}\right\}
$$

Discussion of this Equation, obtained by simple application of some basics concepts of probability theory, allows us to understand the effect of phase fluctuations on the diffraction pattern. It is to be noted that factor $(\sin \Omega)^{2} / \Omega^{2}$ prevents the second term in parenthesis from being exactly +1 or -1 , as 
in the deterministic case (Eq 8). Therefore, the maximum value of the entire parenthesis is less than 2 , and the minimum is not zero and they give rise to maximum and minimum average intensities, $\langle\mathrm{I}(\mathrm{P})\rangle_{\max }$ and $\langle\mathrm{I}(\mathrm{P})\rangle_{\min }$ given by

$$
\begin{aligned}
& <\mathrm{I}(\mathrm{P})>_{\max }=2 \mathrm{~A}^{2}\left\{1+(\sin \Omega)^{2} / \Omega^{2}\right\} \\
& <\mathrm{I}(\mathrm{P})>_{\min }=2 \mathrm{~A}^{2}\left\{1-(\sin \Omega)^{2} / \Omega^{2}\right\}
\end{aligned}
$$

This means that phase fluctuations reduce the values of the intensity in the maxima and raise the values in the minima. Note, in addition, that the presence of fluctuations does not change the positions of the points where maxima or minima arise; in other words fringe positions do not change.

To describe the quality of the fringes one generally uses "fringe visibility", defined as

$$
V=\frac{I_{\max }-I_{\min }}{I_{\max }+I_{\min }}
$$

This definition can be here used for "average intensities". From Eqs 17 and 18 one gets

$$
\mathrm{V}=(\sin \Omega)^{2} / \Omega^{2}
$$

This result clearly shows us the effect of the phase fluctuations. The effect can be very small, for small $\Omega$ (eg for $\Omega=\pi / 10 \mathrm{~V}=0.97$ ) but rapidly increases with $\Omega$, for $(\Omega=\pi / 2, V=0.4)$ reaching the dramatic result of vanishing visibility for $\Omega$ approaching $\pi$. It is also interesting to note the undulating behaviour of $(\sin \Omega)^{2} / \Omega^{2}$, for instance when $\Omega=3 \pi / 2$ its value is 0.045 .

The $\Omega=\pi$ case can be used as a simple model to explain the behaviour of natural light which does not give fringes but simply addition of energies (the first two terms of Eq 15). This is commonly referred to as incoherence.

Another more realistic example, which will be useful in the sequel, is that of phase fluctuations with Gaussian distribution. Let us now consider the case of phases still uncorrelated but having identical Gaussian distributions

$$
p\left(\varphi_{1}\right)=p\left(\varphi_{2}\right)=\frac{1}{\sigma \sqrt{2 \pi}} e^{-\varphi_{1}^{2} / 2 \sigma^{2}}
$$

To evaluate the average of $\cos \varphi_{1}$, defined by

$$
<\cos \varphi_{1}>=\frac{1}{\sqrt{2 \pi} \sigma} \int_{-\infty}^{\infty} \cos \varphi e^{-\varphi^{2} / 2 \sigma^{2}} \mathrm{~d} \varphi
$$

it is useful to pass to the exponential definition of the cosine. This evaluation, also useful in the sequel, is carried out in appendix $\mathrm{A}$. One obtains

$$
\begin{aligned}
& <\cos \varphi_{1}>=<\cos \varphi_{2}>=e^{-\sigma^{2} / 2} \\
& <\sin \left(\varphi_{1}-\varphi_{2}\right)>=0
\end{aligned}
$$

Introduction of these results into Eq.11 and then substitution into Eq.9 gives 
All the results of the preceding case can therefore be extended to the case of Gaussian probability provided that the exponential term $e^{-\sigma^{2}}$ replaces the term $(\sin \Omega)^{2} / \Omega^{2}$. As a consequence of the exponential term, the decay in the Gaussian case is more rapid than in the equiprobable case and does not have oscillating behaviour. For instance when $\sigma=\pi / 2$ the fringe visibility is $\mathrm{V}=0.085$, for $\sigma=\pi \quad \mathrm{V}=510^{-5}$, and for $\sigma=3 \pi / 2 \quad \mathrm{~V}=210^{-10}$.

We can conclude this section by observing that knowledge of a little probability theory can help to solve the problem of independent phase fluctuations.

\section{A MORE DIFFICULT PROBLEM REgUIRING A STATISTICAL APPROACH}

The hypothesis of phase fluctuations at the two holes independent of one another is quite simple, and very useful in many applications, but does not describe a general and more common situation. In general the phase fluctuations at the two holes can be correlated. For instance, if a wave crosses a few meter path in the atmosphere and the two holes are not too far from one another the fluctuations at one point are somehow related to those at the other point.

Starting again from Eq 9 we cannot proceed as in the case of independent fluctuations with $<\cos \left[\varphi_{1}\right.$ $\left.-\varphi_{2}-\mathbf{k}\left(\mathrm{r}_{1}-\mathrm{r}_{2}\right)\right]>$, but we must stop after $\mathrm{Eq} \mathrm{10}$. In Eq.10 it is useful to rewrite the trigonometric functions in complex form

$$
\begin{aligned}
& <\cos \left(\varphi_{1}-\varphi_{2}\right)>=<\left\{\mathrm{e}^{\mathrm{i}\left(\varphi_{1}-\varphi_{2}\right)}+\mathrm{e}^{-\mathrm{i}\left(\varphi_{1}-\varphi_{2}\right)}\right\} / 2> \\
& <\sin \left(\varphi_{1}-\varphi_{2}\right)>=<\left\{\mathrm{e}^{\mathrm{i}\left(\varphi_{1}-\varphi_{2}\right)}-\mathrm{e}^{-\mathrm{i}\left(\varphi_{1}-\varphi_{2}\right)}\right\} / 2 \mathrm{i}>
\end{aligned}
$$

The problem now is to evaluate the average of the complex exponentials.

Let us assume that $\varphi_{1}$ and $\varphi_{2}$ have both Gaussian distribution (with variance $\sigma^{2}$ ). This assumption is a very realistic one because phase fluctuations are generally the result of many small path effects and therefore, for the Central Limit Theorem, obey a Gaussian pdf. Consequently their difference $\mathrm{y}=\varphi_{1}-\varphi_{2}$ is Gaussian with variance $\Phi^{2}$ given by

$$
\Phi^{2}=\left\langle\mathrm{y}^{2}>-\langle\mathrm{y}\rangle^{2}=\left\langle\left(\varphi_{1}-\varphi_{2}\right)^{2}>-<\left(\varphi_{1}-\varphi_{2}\right)^{2}\right.\right.
$$

By developing the square, and applying the same rules to averages and sums as before, one obtains

$$
\Phi^{2}=<\varphi_{1}^{2}>+<\varphi_{2}{ }^{2}>-2<\varphi_{1} \varphi_{2}>-<\varphi_{1}>^{2}-<\varphi_{2}>^{2}+2<\varphi_{1}><\varphi_{2}>
$$

Without loss of generality let us assume that the average phases are zero. The last three terms are therefore zero and Eq 24 reduces to.

$$
\Phi^{2}=<\varphi_{1}^{2}>+<\varphi_{2}{ }^{2}>-2<\varphi_{1} \varphi_{2}>
$$

The first two terms are the variances of the phase fluctuations while the third term, the average of the "product", represents the correlation term. In statistical language the last term is the phase 
correlation function at two points on the wavefront impinging on the holed screen as a function of the point separation; in our case the separation between the holes. (Quantity $\Phi^{2}$, Eq.28, is called the structure function of the phase). The probability function of difference $y$ is therefore given by:

$$
p(y)=\frac{1}{\Phi \sqrt{2 \pi}} e^{-y^{2} / 2 \Phi^{2}}
$$

which also takes into account correlation. The problem is now reduced to evaluating the averages of the exponents exp(iy) and exp(-iy) to be introduced into Eq.s 26 and 27. This is immediately done by applying the results of Appendix A. One obtains

$$
\begin{aligned}
& <\cos \left(\varphi_{1}-\varphi_{2}\right)>=e^{-\Phi^{2} / 2} \\
& <\sin \left(\varphi_{1}-\varphi_{2}\right)>=0
\end{aligned}
$$

The average intensity on a point $P$ of the far screen is obtained by introducing Eq.s 32 and 33 into Eq. 10 and then the result into Eq.9.

$$
<\mathrm{I}(\mathrm{P})>=\mathrm{A}_{1}{ }^{2}+\mathrm{A}_{2}{ }^{2}+2 \mathrm{~A}_{1} \mathrm{~A}_{2} \cos \left[\mathrm{k}\left(\mathrm{r}_{1}-\mathrm{r}_{2}\right)\right] e^{-\Phi^{2} / 2}
$$

The equation is formally similar to that of the previous case of uncorrelated fluctuations, and in fact it reduces to Eq. 25 in the case of absence of correlation. However it has a more complete meaning because it also includes correlation. By recalling that the variance of both phases is $\sigma^{2}$, from Eq.30 one can write

$$
\Phi^{2}=\sigma^{2}+\sigma^{2}-2<\varphi_{1} \varphi_{2}>=2 \sigma^{2}\left(1-<\varphi_{1} \varphi_{2}>/ \sigma^{2}\right)
$$

The quantity

$$
\mathrm{r}=\left\langle\varphi_{1} \varphi_{2}>/ \sigma^{2}\right.
$$

is called the correlation coefficient, and Eq 35 becomes

$$
\Phi^{2}=2 \sigma^{2}(1-r)
$$

Without more knowledge of the correlation coefficient, which can be acquired by the theory of random functions, it is difficult to comment on these results. One can here obtain some results by looking at the fringe visibility. With the simplifying hypothesis of equal amplitudes, A, one has

$$
<\mathrm{I}(\mathrm{P})>=2 \mathrm{~A}^{2}\left\{1+\cos \left[\mathrm{k}\left(\mathrm{r}_{1}-\mathrm{r}_{2}\right)\right] e^{-\Phi^{2} / 2}\right\}
$$

and the visibility:

$$
\mathrm{V}=e^{-\Phi^{2} / 2}
$$

From this result and Eq.37 one can see that the presence of a correlation in the phase fluctuations can "help" in getting higher visibility with respect to the case of no correlation. If we were aware of the theory of partial coherence and random functions we would recognize that $e^{-\Phi^{2} / 2}$ is the correlation (covariance) function of the fluctuating complex signal of the field at the two points corresponding to the holes. 


\section{OTHER CASES}

Let us now try to understand what happens if the amplitude fluctuates. There are a number of possible combinations between amplitude and phase fluctuations:

1 - Amplitude only fluctuates, and

1.a Amplitude fluctuations at the two points are independent of one another, or

1.b Amplitude fluctuations at the two points are correlated.

2- Both amplitude and phase fluctuates independently

2.a Amplitude fluctuations are uncorrelated and also phase fluctuations are uncorrelated

2.b Amplitude fluctuations are uncorrelated but phase fluctuations are correlated

2.c Amplitude fluctuations are correlated and phase fluctuations are uncorrelated

2.d Amplitude fluctuations are correlated and phase fluctuations are correlated

3- Both amplitude and phase fluctuate in a correlated way.

One could ask if so many possibilities correspond to real cases found in practice. The answer is yes. For instance case 1 represents the case of a signal from an adaptive system operating horizontally in the atmosphere which achieves a complete correction of phase fluctuations, in the two cases of: 1.a) two points (holes in our case) far from one another ( $>$ few $\mathrm{mm}$ ) or $1 . \mathrm{b}$ ) very near (eg $1 \mathrm{~mm}$ or less). Case 2 can represent different situations corresponding to waves after propagation through random media or the case of an adaptive system in the atmosphere where the phase correction is incomplete. Case 3, the most difficult one, is the general case of propagation through random media, in the case of coherent scattering.

It is not the purpose of the present paper to analyze in detail all possible situations listed above. We want here to stress the need for knowledge of statistical optics and therefore will consider only the simplest case 1.a): uncorrelated amplitude fluctuations.

To find the pattern of the far screen one still has to find the average intensity. Starting again from mother equation (Eq 7) one has to determine the average intensity with respect to amplitude, and obtains

$$
\mathrm{I}(\mathrm{P})=\left\langle\mathrm{A}_{1}^{2}>+<\mathrm{A}_{2}^{2}>+2<\mathrm{A}_{1} \mathrm{~A}_{2}>\cos \left[\varphi_{1}-\varphi_{2}+\mathrm{k}\left(\mathrm{r}_{1}-\mathrm{r}_{2}\right)\right]\right.
$$

which, for uncorrelated and statistically equal amplitude fluctuations, and equal phases, becomes

$$
\left.\mathrm{I}(\mathrm{P})=2<\mathrm{A}^{2}>+2<\mathrm{A}\right\rangle^{2} \cos \left[\mathrm{k}\left(\mathrm{r}_{1}-\mathrm{r}_{2}\right)\right]=2\langle\mathrm{~A}\rangle^{2}\left\{<\mathrm{A}^{2}>/<\mathrm{A}>^{2}+\cos \left[\mathrm{k}\left(\mathrm{r}_{1}-\mathrm{r}_{2}\right)\right]\right\}
$$

In this expression, in addition to average amplitude square $\langle A\rangle^{2}$, term $\left\langle A^{2}\right\rangle \mid\langle A\rangle^{2}$, the second normalized moment (non centered) of amplitude appears. This is an important quantity for characterization of the intensity fringes.

To proceed further it would be now necessary to choose a pdf for the amplitude. As amplitude is a positive quantity, Gaussian or equiprobable distributions are not useful apart from the limiting case of very small fluctuations with respect to the average amplitude. One important distribution for amplitude is the so called log-normal distribution. This means that the logarithm of the amplitude, not the amplitude itself, is normally distributed. Log-normal distribution is found in propagation through random media, in particular atmospheric turbulence, for instance when the medium presents small fluctuations or the path is short enough.

Without entering into the details of amplitude distributions, some results can be obtained directly from Eq.41. First we can note that the first term in the parenthesis, $\left\langle A A^{2}\right\rangle /\langle A\rangle^{2}$, is larger than or equal to one, the equality sign being valid in the limiting case of no fluctuations. This introduces a 
"bias" in the interference pattern and would require some energy considerations. To take into account that total energy needs to remain constant, an additional normalization factor would be necessary. By limiting ourselves to find fringe visibility, a quantity independent of such a normalization factor, we obtain from Eq.41:

42). $\mathrm{V}=\langle\mathrm{A}\rangle^{2} /\left\langle\mathrm{A}^{2}\right\rangle$

Therefore fringe visibility is determined, inversely, by the second moment of the amplitude, which is always larger than one and tends to one only in the case of vanishing fluctuations. As a consequence amplitude fluctuations also alter the visibility of the fringes, and this can be easily understood. On the far screen the maxima, as well as the minima, of the intensity are affected by the fluctuating values of the amplitude, and result in a lowering of the visibility.

In conclusion, concerning Young interferometer, we note that the "mother equation", Eq. 7, allows one to study the average intensity in all cases listed at the beginning of this section, provided that knowledge of probability distributions and corresponding correlations is avallable.

\section{GENERAL CONSIDERATIONS ON STATISTICAL OPTICS AND CONCLUSION}

So far we have examined the simple case of average intensity in the Fraunhofer pattern of a Young interferometer. For most problems the intensity variance at each point is also of importance. To deal with this problem in the case of uncorrelated fluctuations is not a difficult job, provided one is able to evaluate average square intensity. The problem becomes much more difficult, even conceptually, when there is correlation.

Another simple example of statistical optics is the diffraction pattern of an aperture, which however is more difficult because it is impossible to neglect the correlation, even when the correlation area on the aperture is small.

Let us now mention some of all the ensemble of phenomena which go under the name of statistical optics. And here we explicitly neglect inverse problems which require profound knowledge of statistical inference methods.

Dealing with (partially) correlated fields requires more sophisticated statistics, such as coherence theory knowledge (time and space coherence), the concept of analytic signal, as well as the theory of random functions, spectra and some fundamental theorems like Wiener-Kinchine. Often correlation or covariance functions of higher orders are required. For example, the study of propagation in random media (eg scintillation) requires fourth order statistics of interest for intensity fluctuations.

The description of light emission by incoherent (e.g.thermal) and coherent (laser) sources can be made in terms of the probability distribution of intensity and photon counting statistics, respectively. The effect of noise in receiving systems as well as in the photographic process involves statistics. Speckle patterns produced by laser light are a problem in statistical optics.

A number of probability density functions have been used for describing intensity fluctuations of coherent light propagated through random media. A typical example is turbulence in the clear atmosphere where a number of different distributions can be found, depending on the strength of the turbulence and evolving from the lognormal pdf for small fluctuations to the negative exponential in the limit of very strong fluctuations.

Incoherent scattering by atmospheric constituents or multiple scattering in dense media (eg smog and biological tissues) are problems involving statistics.

Statistics can also be done numerically, through Monte Carlo methods of simulation. The methods 
are useful for both propagation through clear atmosphere (coherent scattering) and for scattering through fog or smog and in particular for the above case of dense media.

A description of the average behaviour of optical systems in random media, for instance the locally stationary atmosphere, can be given in terms of (average) Modulation Transfer Function (MTF) of the atmosphere, another statistical quantity.

Many other topics on statistical optics can be found in a number of books devoted to the subject or including general papers on the subject some of which are listed below for information(Ref 3 to 12). The list is not intended to be exhaustive. It includes the recent series of the ICO volume "International Trends in Optics" (Ref 8,9,11,12) where precise chapters are devoted to modern topics of statistical optics.

At this point the answer to the question in the title: "should optics student know statistics?" should be obvious to the Reader.

\section{List of books}

1- A. Consortini: "How much mathematics should optics students know?" in 'Education in Optics' G.B. Altshuler and B. J. Thompson Editors, Proc. SPIE vol1603, 98-108, 1992.

2 - A. Consortini "Diffraction and Fourier Optics: The Mathematics required" in 1995 International Conference on Education in Optics, M. J Soileau Editor, Proc. SPIE 2525, 678-688, 1995.

3 - E.L. O’Neill: "Introduction to Statistical Optics" Addison-Wesley, 1963

4 - J.C. Dainty and R. Shaw, "Image Science", Academic Press, 1974.

5 - A. Ishimaru "Wave Propagation and Scattering in Random Media", Academic Press, 1978.

6 - J.W. Goodman "Statistical Optics", John Wiley and Sons, New York, 1985

7 - S.M. Rytov, Yu A. Kravtsov, V.I. Tatarskii, "Principles of Statistical Radiophysics Vol.4: Wave Propagation in Random Media", Springer-Verlag, 1989.

8 - J.W. Goodman, Editor "International Trends in optics" Academic Press, 1991 (ICO vol 1, see.e.g. Chapters 15, 16, 17, 19, 26, 27).

9 - J. C. Dainty, Editor "Current trends in Optics" Academic Press, 1994 (ICO vol 2, Ch.8, 9 and 21 ).

10- L.Mandel and E.Wolf "Optical Coherence and Quantum Optics" Cambridge Univ. Press 1995

11 - A.Consortini, Editor "Trends in Optics: Research, Developments and Applications, ICO Book 3 Academic Press 1996 (see e.g.Chapters 2, 8, 17,18)

12- T Asakura, Editor "International Trends in Optics and Photonics" ICO IV, Springer, 1999.

\section{APPENDIX A}

In the case of a variable, $x$, having a Gaussian distribution

$$
\mathrm{p}(\mathrm{x})=\frac{1}{\sqrt{2 \pi} \sigma} e^{-x^{2} / 2 \sigma^{2}}
$$

the average cosine is given by:

$$
<\cos x>=\frac{1}{\sqrt{2 \pi} \sigma} \int_{-\infty}^{\infty} \cos x e^{-x^{2} / 2 \sigma^{2}} \mathrm{~d} x
$$

To evaluate this integral it is useful to pass to the exponential definition of the cosine and sine

$$
\cos x=\frac{e^{i x}+e^{-i x}}{2} \quad \sin x=\frac{e^{i x}-e^{-i x}}{2 i}
$$


Introduction of A3 into A2 shows that one has to evaluate

$$
<\cos x>=\frac{1}{2}\left\lfloor\frac{1}{\sqrt{2 \pi} \sigma} \int_{-\infty}^{\infty} e^{i x} e^{-x^{2} / 2 \sigma^{2}} \mathrm{~d} x+\frac{1}{\sqrt{2 \pi} \sigma} \int_{-\infty}^{\infty} e^{-i x} e^{-x^{2} / 2 \sigma^{2}} \mathrm{~d} x\right\rfloor
$$

Let us start with the first integral in the brackets. In the exponent, it is useful to add and remove a constant term, $b$, in such a way that the part containing $x$ becomes part of a perfect square. This can be obtained by letting

$$
i x-x^{2} / 2 \sigma^{2}=-(a x-b)^{2}+b^{2}=-a^{2} x^{2}+2 a b x
$$

from which one has

$$
a=1 / \sqrt{2} \sigma
$$

$$
b=i / 2 a=i \sigma / \sqrt{2}
$$

and the integral becomes:

$$
I_{1}=\frac{1}{\sqrt{2 \pi} \sigma} \int_{-\infty}^{\infty} e^{i x} e^{-x^{2} / 2 \sigma^{2}} \mathrm{~d} x=\frac{1}{\sqrt{2 \pi} \sigma} \int_{-\infty}^{\infty} e^{-[x / \sqrt{2} \sigma-i \sigma / \sqrt{2}]^{2}} e^{-\sigma^{2} / 2} \mathrm{~d} x
$$

The change of variable

$$
x / \sqrt{2} \sigma-i \sigma / \sqrt{2}=y
$$

gives

A10)

$$
I_{1}=\frac{e^{-\sigma^{2} / 2}}{\sqrt{\pi}} \int_{-\infty}^{\infty} e^{-y^{2}} \mathrm{~d} x=e^{-\sigma^{2} / 2}
$$

where the value $\sqrt{\pi}$ of the integral, representing the well known value of the error function $(\operatorname{Erf}(\infty)=\sqrt{\pi})$, has been introduced.

It is easy to show that also the second integral of Eq A4

$$
I_{2}=\frac{1}{\sqrt{2 \pi} \sigma} \int_{-\infty}^{\infty} e^{-i x} e^{-x^{2} / 2 \sigma^{2}} \mathrm{~d} x
$$

gives rise to the same result. It suffices to replace $i$ by $-i$ in all the procedure to obtain:

$$
I_{1}=I_{2}
$$

and finally, from Eq. A4 and A3:

$$
<\cos x>=e^{-\sigma^{2} / 2} \quad \text { and } \quad<\sin x>=0
$$

